\title{
Study of dioxin removal from sintering flue gas
}

\author{
Tingyu Zhu*, Yangyang Guo, Feng Qi, Xiaomiao Yan
}

Abstract-Dionxin is difinetly harmful to human body, and large part of dioxin is produced from sintering processes, the distribuition of dionxin with sintering temperature is shown in Figure 1, so the control for dioxin in sintering process is quite essential. One of the mosy common ways for dioxin removal is adsorption by activated carbon, while the real dioxin gas is much complicated and quite hard to generate in laboratory, so the researches for dioxin removal by activated carbon are mostly related to the plot scale test. The AC consumption and the dioxin removal efficencies can be got from these studies, while the mechanism for dioxin removal by activated carbon can not been analysed from these scale experiments. In order to inveatigate the mechnism of dioxin removal on carbon, our research work studied a series chlorohydrocarbon's adsorption on activated carbon, the adsorption capacity of dioxin is caculated based on our experimental results and the desorption species are also deeply studied by TPD-MS methord, according to the interactions betwwen dioxin-like componds and carbon surface (including the oxygen functional groups), the mechanism of dioxin removal by activated carbon is illustrated in this paper.

Keywords - dioxin, activated carbon, mechanism, sintering

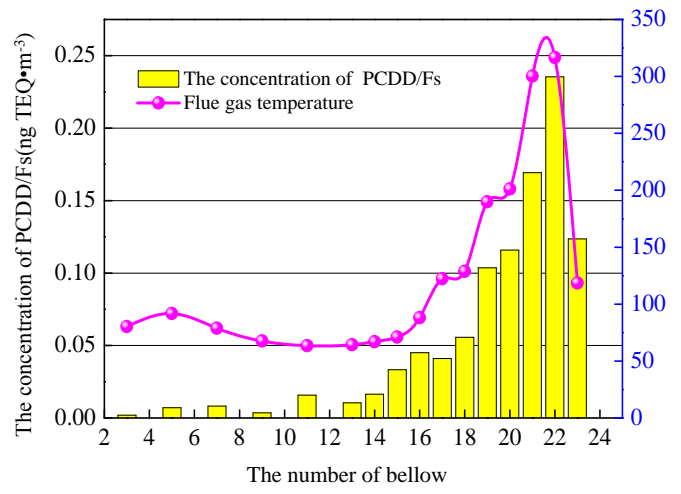

Figure 1. The variation of $\mathrm{PCDD} / \mathrm{Fs}$ concentration with sinterring temperatures

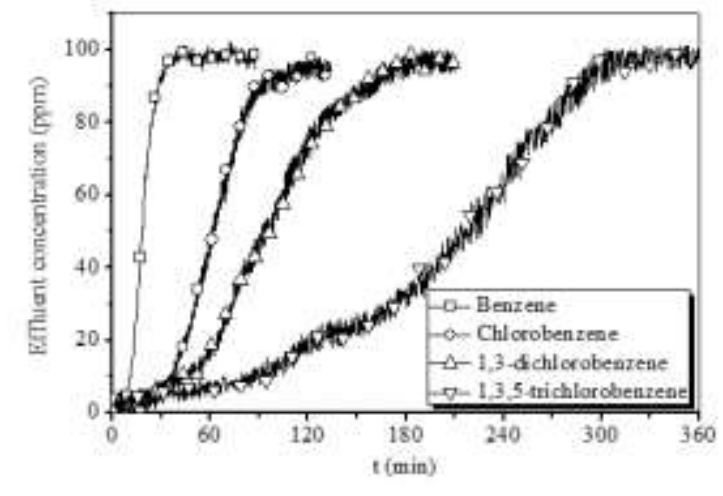

Figure 2. The breakthrough curves of chlorohydrocarbon on AC

Tingyu Zhu (Correspongding author)

Institute of Process Engineering, Chinese Academy of Sciences

China

Yangyang Guo(Author)

Institute of Process Engineering, Chinese Academy of Sciences

China

Feng Qi (Author)

Institute of Process Engineering, Chinese Academy of Sciences

China

Xiaomiao Yan (Author)

Institute of Process Engineering, Chinese Academy of Sciences

China 